\title{
SOFIA Water Vapor Monitor Design
}

\author{
R. Cooper', T. L. Roellig' ${ }^{2}$, L. Yuen', B. Shiroyama', A. Meyer ${ }^{3}$ \\ ${ }^{1}$ TechnoScience Corporation, Palo Alto, CA USA \\ ${ }^{2}$ NASA Ames Research Center, Moffett Field, CA USA \\ ${ }^{3}$ USRA, NASA Ames Research Center, Moffett Field, CA USA
}

\begin{abstract}
The SOFIA Water Vapor Monitor (WVM) is a heterodyne radiometer designed to determine the integrated amount of water vapor along the telescope line of sight and directly to the zenith. The basic technique that was chosen for the WVM uses radiometric measurements of the center and wings of the $183.3 \mathrm{GHz}$ rotational line of water to measure the water vapor. The WVM reports its measured water vapor levels to the aircraft Mission Controls and Communication System (MCCS) while the SOFIA observatory is in normal operation at flight altitude. The water vapor measurements are also available to other scientific instruments aboard the observatory. The electrical, mechanical and software design of the WVM are discussed.
\end{abstract}

\section{Introduction}

The SOFIA Water Vapor Monitor (WVM) is responsible for taking measurements so that the precipitable amount of water vapor, both along the telescope line of sight and directly to the zenith, is known while the SOFIA aircraft is at normal operational altitudes. The accuracy of the measured precipitable water vapor must be 2 microns or better, 3 sigma, and measured at least once a minute. This data is used to correct the astronomical infrared data obtained by the telescope and will also be used in the algorithm that determines successful SOFIA observatory flight hours for contractual purposes. The WVM reports the measured water vapor levels in the zenith direction to the aircraft Mission Controls and Communication System (MCCS) once every 15 seconds while the SOFIA observatory is in normal operation at flight altitude. The MCCS then uses the data to derive the amount of water vapor along the line of sight of the telescope.

The basic technique that was chosen for the WVM uses radiometric measurements of the center and wings of the 183.3 $\mathrm{GHz}$ rotational line of water to measure the water vapor level along a line of sight between the WVM and the top of the earth's atmosphere. In order to measure the $183.3 \mathrm{GHz}$ line of water to determine the water vapor overburden, the WVM uses a double sideband heterodyne radiometer that observes in six frequencies offset from the $183.3 \mathrm{GHz}$ line center. These frequency bands are shown in Figure 2 and are listed in Table 1. The sixth channel covers the entire range of the other five channels and is used for diagnostic purposes as well as in water vapor determination. The amount of water vapor in the zenith direction is determined by calculation from measurements along a line of sight fixed with respect to the aircraft frame that is at a $40^{\circ}$ elevation angle relative to the horizon while in level flight. The WVM measurements must be corrected for the true aircraft roll and pitch angles during the measurements. The aircraft roll and pitch angles are provided to the WVM by the aircraft flight system autopilot, which can measure these parameters to better than $0.1^{\circ}$. The radiometer views the sky through a custom pressure window transparent to $183 \mathrm{GHz}$ radiation that is mounted in a window plug in the upper deck of the Boeing 747SP.

The Water Vapor Monitor hardware consists of three physically distinct hardware items: the Radiometer Assembly (RHD), the WVM IF Converter (IFC), and the WVM Computer (WVMCPU) as shown in Figure 1. 


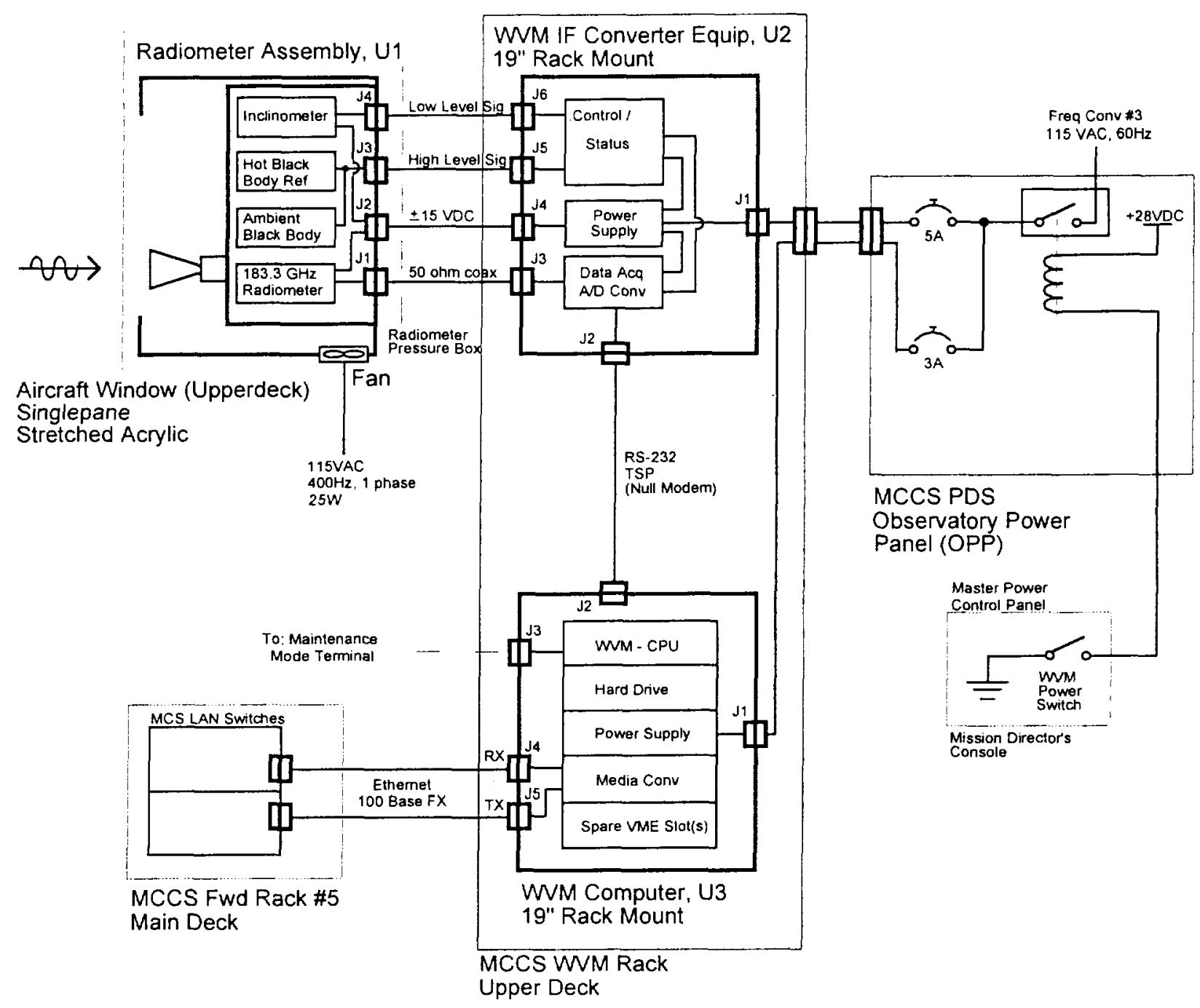

Figure 1. WVM system block diagram.

\section{WVM Water Vapor Derivation Algorithm}

The water vapor overburden is determined by fitting the measured signal in the six radiometer frequency bands to a simplified model of the atmosphere. In order to derive the accuracy requirements for the various measurements that are taken by the WVM, we first have to model the emission from the atmosphere into each of the six WVM frequency bands. This involves solving the equations of radiative transfer, starting at the top of the atmosphere down to the aircraft along a $40^{\circ}$ elevation line of sight. In our modeling we started at an altitude of $29.0 \mathrm{~km}$ (13.7 mbar) in the US Standard Atmosphere and divided the atmosphere into layers $100 \mathrm{~m}$ thick. We can safely assume that water vapor emission from altitudes above $29 \mathrm{~km}$ is negligible. In order to facilitate analysis using standard radio frequency analysis tools, we have expressed our modeling results in terms of brightness temperature in each of the six radiometer frequency bands. The results for the US Standard Atmosphere are given in Table 1 below. At $12.5 \mathrm{~km}(41,000 \mathrm{ft})$ the US Standard Atmosphere has a water vapor overburden to the zenith of 7.09 precipitable microns. 


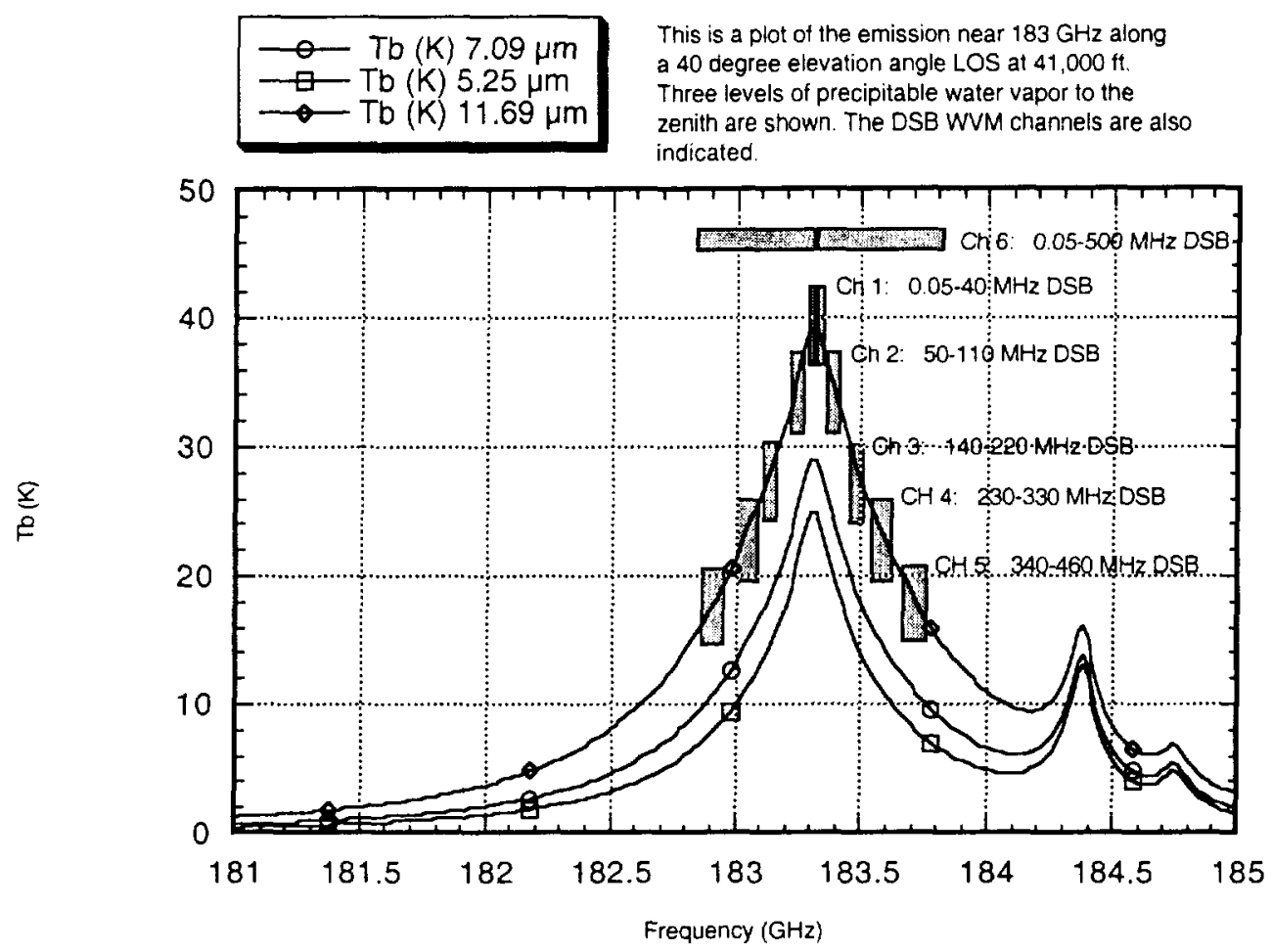

Figure 2. Water emission line near $183 \mathrm{GHz}$

Table 1

Line Brightness Temperatures in the US Standard Atmosphere

\begin{tabular}{|c|c|}
\hline Channel Number & $183 \mathrm{GHz}$ Line Brightness Temperature (K) \\
\hline 1 & 28.481 \\
\hline 2 & 24.172 \\
\hline 3 & 18.389 \\
\hline 4 & 14.433 \\
\hline 5 & 11.001 \\
\hline 6 & 21.780 \\
\hline
\end{tabular}

With the US Standard Atmosphere as a point of departure, we added variability into the parameters of pressure and temperature to those atmospheric layers located between the flight altitude and $17.5 \mathrm{~km}(81 \mathrm{mbar})$. This then allows us to evaluate the effects on the water vapor determination of not knowing the exact values of the temperature and pressure, either because our aircraft sensors are not perfectly accurate, or due to the deviations between the real atmosphere and the US Standard Atmosphere that we are trying to measure. We only added this variability below $17.5 \mathrm{~km}$ since the stratosphere always agrees quite well with the Standard Atmosphere at higher altitudes. It should also be noted that even if this approximation was not strictly true, the relative lack of water vapor above $17.5 \mathrm{~km}$ would make this effect minor.

In a similar way we also added variable water vapor level to the atmospheric levels below $17.5 \mathrm{~km}$. According to Haas and Pfister (1998), this is the most realistic way to model real changes in the atmospheric water vapor content. This modeling was then used to evaluate the WVM sensitivity requirements to derive the water vapor overburden to the required accuracy. Some examples of this modeling are shown below for the aircraft at a flight altitude of $12.5 \mathrm{~km}$ $(41,000 \mathrm{ft})$. The variation of the brightness temperature in each of the WVM channels with water vapor is given in Figure 3 below. It can be seen that even with the highest levels of water vapor, the lines are still not optically thick. 
Varlation with water vapor

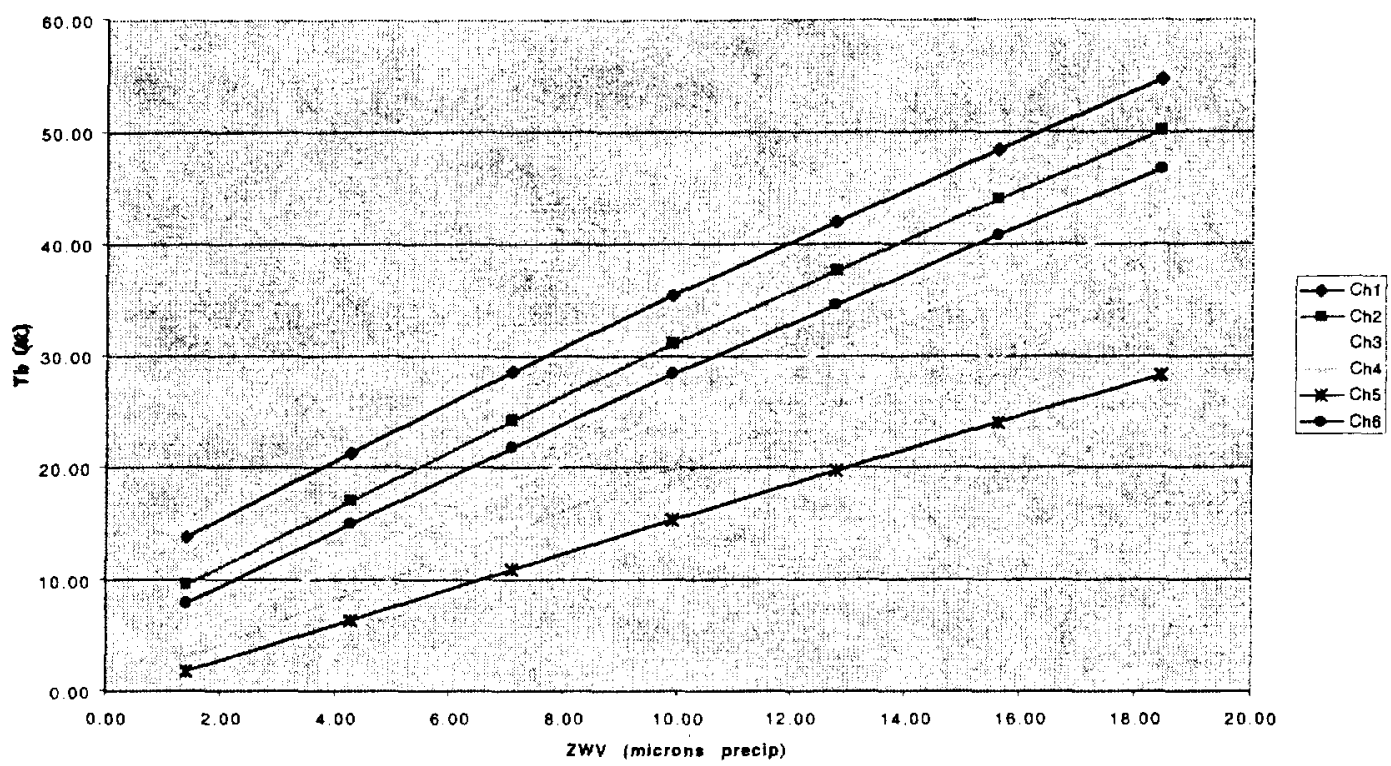

Figure 3. The change in the measured brightness temperature in each of the WVM frequency channels with change in water vapor overburden. The water vapor levels are to the zenith. A ZWV value of $7.09 \mu \mathrm{m}$ corresponds to the US Standard Atmosphere above $41,000 \mathrm{ft}$.

Ideally, one would take the measured WVM radiometer data, together with the aircraft roll angle, pitch angle, outside air temperature, and outside air pressure measurements and invert the radiative transfer model given above to determine the water vapor overburden. Unfortunately, this is too computationally intensive for the nearly real-time WVM data output specified in the WVM requirements. Instead, the following process is used to derive the water vapor level.

The measured voltage signal from each radiometer channel is first converted to measured brightness temperature using the two blackbody reference targets within the WVM together with transfer and efficiency functions measured in the laboratory. This then allows the determination of the brightness temperature of any emission entering the radiometer frequency channels.

In order to speed up the water vapor overburden derivation, the water line emission is modeled simply as coming from two layers, one layer consisting of all altitudes above a pressure of $81 \mathrm{mbar}$, and the other layer consisting of the altitudes between this level and the aircraft. The boundary between these two layer corresponds to an altitude of 17.5 $\mathrm{km}$ in the US Standard Atmosphere. In atmospheric modeling the pressure is a more basic independent parameter than altitude, so the water vapor derivation algorithm is a function of pressure instead of altitude. The algorithm assumes that the emission in the upper layer is constant in time and location. For the lower layer, its absorption of the emission above it and its own emission is assumed to be a function of air temperature and pressure outside of the aircraft multiplied by the average water vapor level in the layer.

Water vapor is determined by using the radiometer data together with the ambient air temperature and pressure to determine the total water vapor overburden, which is the sum of the average amount of water vapor in the lower layer and the constant water vapor content of the upper layer. This approximation breaks down at higher water vapor levels, but is sufficiently accurate for zenith water vapor overburden levels within the WVM requirement range ( $4 \mu \mathrm{m}$ to 20 $\mu \mathrm{m})$. In comparing the water vapor determined with this algorithm to the full-blown radiative transfer calculation we find that the maximum error is 0.05 microns over the requirements range. Once the water vapor overburden to the zenith is determined, it is a simple matter for the MCCS to calculate the water vapor along the telescope line of sight since the telescope elevation angle is known to a very high accuracy. 


\section{The WVM Radiometer Head (RHD)}

The Radiometer Head contains an antenna that views the sky, two calibrated reference targets (one heated and one ambient temperature microwave black body), an RF switch, a mixer, a local oscillator, an IF amplifier, and an inclinometer. All of these components are mounted together on a baseplate and are attached to the inner surface of the aircraft fuselage so that the antenna can observe the sky through a microwave-transparent window. Power to the entire RHD is provided by power supplies in the WVM IF Converter Box.



Figure 4. Radiometer Head.

The WVM antenna is mounted fixed to the aircraft frame with the WVM line of sight at a nominal $40^{\circ}$ elevation angle while the aircraft is in level flight. The antenna itself employs a quasi-optical design with a microwave lens that feeds a feed horn. In order that the WVM not be unduly sensitive to $183.3 \mathrm{GHz}$ emission from the ground or lower atmosphere, an antenna beam width of less than $2^{\circ}$ with minimal side-lobes is necessary. The antenna chosen was a quasi-optical lens-horn combination with a roughly Gaussian beam pattern (GOA) and a full-width-half-maximum diameter of $0.87^{\circ}$. A sub-harmonic mixer mounted directly behind the feed horn mixes the $183.3 \mathrm{GHz}$ radiation down to a bandwidth of 1 $\mathrm{GHz}$ with the radiometer operating in double sideband mode. The sub-harmonic mixer is fed by a phase-locked 91.65 $\mathrm{GHz}$ local oscillator. The intermediate frequency signal out of the mixer is amplified by an RF amplifier with a frequency range of $100 \mathrm{kHz}$ to $500 \mathrm{MHz}$ before it is sent to the IF Converter Box.

The Water Vapor Monitor RHD also includes an inclinometer that can be used to reference the Water Vapor Monitor line of sight to the aircraft autopilot reference systems. Since this inclinometer works by measuring the position of a fluid, it is not suitable for accurate operation while in the air. It will instead be used for pre-flight ground calibration of the WVM line of sight to the aircraft autopilot signal. While in flight the WVM will derive its true elevation angle from the roll and pitch angle data provided by the aircraft flight management system. 
Since the WVM operates as a radiometer, accurate gain stability is important. In order to achieve this stability two reference blackbody targets are used to periodically insert a stable signal into the radiometer signal chain as follows. After the radiometer has viewed the sky for 5 seconds, a motor-driven mirror redirects the view of the radiometer towards the blackbody calibrators. After the radiometer has viewed the ambient blackbody for 5 seconds, the ambient blackbody is moved out of the field of view, exposing the hot blackbody to the radiometer. Therefore, over a 15 second period the radiometer observes the sky for 4 seconds, an ambient temperature blackbody target for 4 seconds, and a heated blackbody target for 4 seconds. The one second difference at each viewing position allows time for the mirrors to move and the radiometer signal to settle. The temperatures of the two back body targets are not actively controlled in a feedback loop. Instead, temperature sensors on the targets are used in the WVM radiometer for gain monitoring. Additional gain calibration is provided by a temperature sensor mounted to the RF amplifier in the RHD, which is the first $R F$ gain stage and as such has the greatest effect on the system signal-to-noise ratio.

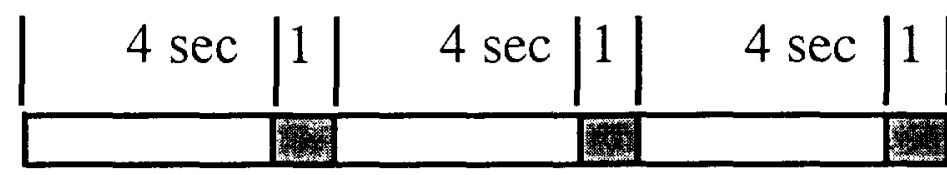

\section{Sky $\quad$ Amb BB Hot BB}


Figure 5. Timing diagram for elliptical mirror and black body unit.

Finally, it should be noted that the WVM window must be absolutely dry while the WVM is in operation. Even the slightest condensation haze on the window will swamp the $\sim$ few microns of integrated atmospheric water vapor along the WVM line of sight.

\section{The WVM IF Converter Box (IFC)}

The IF Converter Box consists of IF filters, IF power splitters, IF amplifiers, IF power meters, analog amplifiers, A/D converters, a $68 \mathrm{HC} 11$ micro-controller, and a RS-232 serial interface driver. These electronics are mounted in a 19" rack cabinet located near the RHD and are connected through cables to both the RHD and the Water Vapor Monitor Host CPU. The software (firmware) that controls the timing of the A/D converters, the movements of the mirror and the ambient blackbody, and the serial communications with the WVM Control Section Software (CSS) is referred to as the Data Acquisition Software (DAS) and resides on the $68 \mathrm{HCl} 1$ board.

The RF signal from the RHD enters the IFC and is first routed to a 1:6 power splitter. The signals in each of the six channels are fed into either low-pass or band-pass RF filters and then further amplified with separate RF amplifiers. Each channel's amplified and filtered signal then goes into an individual RF power detector circuit that uses a tunnel diode as the detection component. The DC output from each diode is further amplified, filtered and then digitized. 


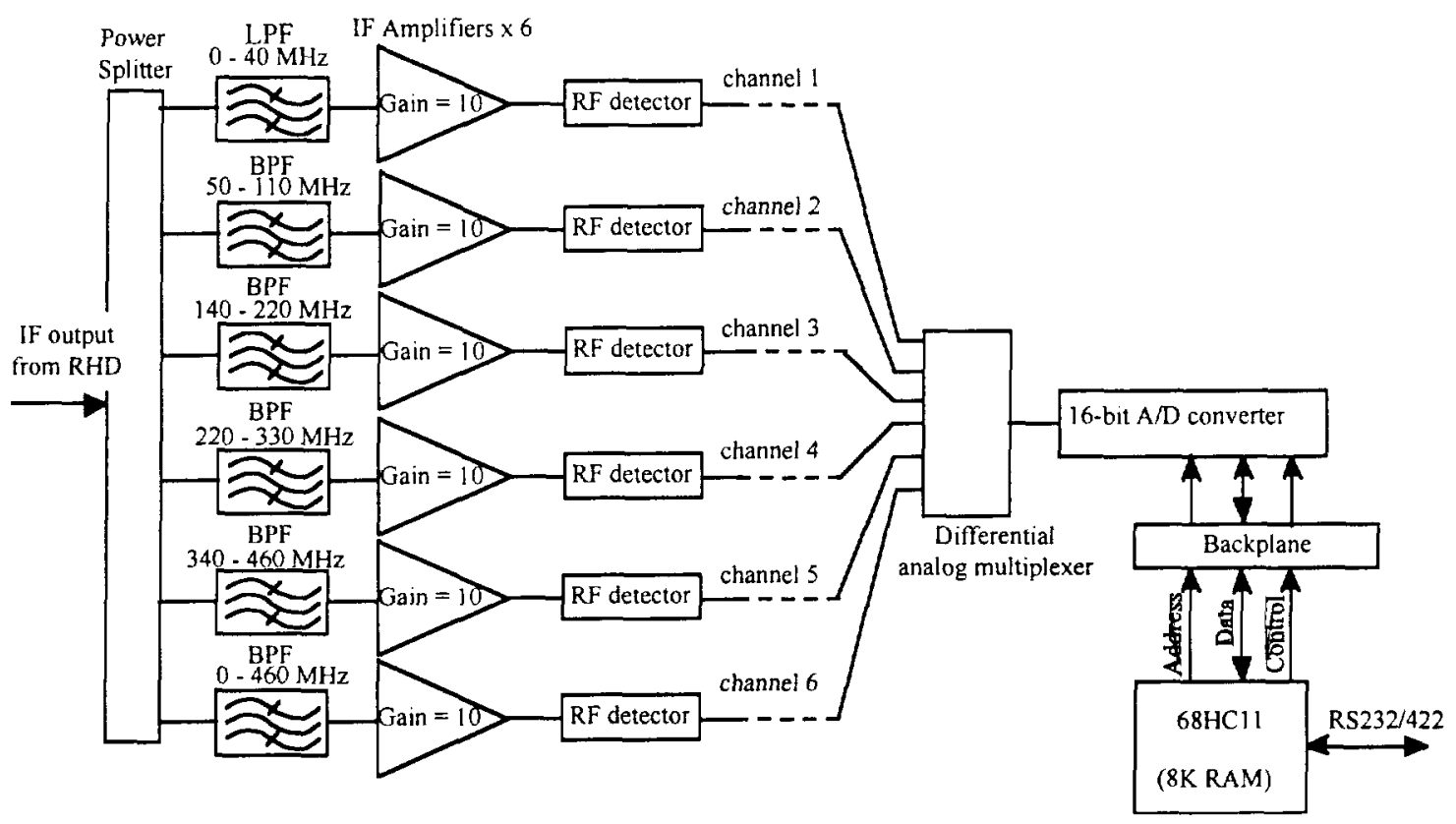

Figure 6. IF channels

The six channels of radiometer data plus two additional channels of stable reference voltage signals (not shown in Figure 6 , they are internal to the A/D converter) are sent to a multiplexed A/D system and are 16-bit digitized, with each channel being sampled every $100 \mathrm{msec}$. A second multiplexed A/D system collects 32 channels of 12 -bit data, denoted as ancillary data, for use in fault detection and signal calibration. The timing and data collection for both A/D systems is controlled by a $68 \mathrm{HCl} 1$ microprocessor, which also handles communications with the WVMCPU over an RS232 serial line.

Three power supplies located in the IFC convert the $120 \mathrm{VAC}$ aircraft power into $+5 \mathrm{~V}$ digital, $\pm 15 \mathrm{~V}$ high-current, and $\pm 15 \mathrm{~V}$ low-noise DC power for use in both the IFC and RHD.

\section{The WVM Host CPU (WVMCPU)}

The Water Vapor Monitor CPU executes the Control Section Software (CSS) that converts the radiometer measurements to microns of precipitable water in the zenith direction and communicates with the MCCS. These electronics are located in the same 19" rack as the WVM IFC and are connected to the IFC through an RS-232 serial line, as shown in Figure 1. The WVMCPU communicates with the SOFIA MCCS through a fiber optics Ethernet connection.

Physically the WVMCPU consists of a Themis single board computer which is based on an $333 \mathrm{MHz}$ UltraSPARC processor, a hard disk, a media converter board, and a power supply that are mounted in a VME backplane in an Aircraft Transport Rack (ATR) located near the 19" rack that holds the IFC. Solaris is used as the WVMCPU operating system. A command was appended to the Solaris startup sequence so that the CSS is launched automatically after the WVMCPU is booted up.

\section{Data Acquisition Software (DAS)}

The Data Acquisition Software operates a $68 \mathrm{HC} 11$ microcontroller running at $3.68 \mathrm{MHz}$ and resides in an $8 \mathrm{~K}$ EEPROM located on the $68 \mathrm{HCl} 1$ board. The DAS controls the timing of the A/D conversions, co-adding of data, $\mathrm{A} / \mathrm{D}$ channel 
selection, mirror movement, blackbody selection, and transmission of data to the CSS.

The DAS software collects digitized radiometer measurements ( 6 radiometer channels, 2 reference channels) and housekeeping data ( 32 channels) and sends them to the CSS at one second intervals via a serial line for processing. Operation of the DAS is controlled by commands sent by the CSS over the serial line. The DAS software is interruptdriven, realtime software written in $68 \mathrm{HC} 11$ assembly language. Hardware timers and interrupts allow data collection to proceed at precisely 12.5 millisecond intervals. After 10 samples have been co-added for each channel $(10 \times 8 \times 12.5$ milliseconds $=1.0$ second), the DAS will transmit the data to the CSS. In the unlikely event that the DAS hangs up, a watchdog timer will automatically restart the DAS so operator action is not needed.

The timing control of the A/D conversions, mirror switch operations, blackbody selection, and management of the coadding the data is performed by the data acquisition interrupt service routine which is programmed in the hardware to execute every 12.5 milliseconds. Each execution of the acquisition routine resets the interrupt to execute in 12.5 milliseconds, reads the output of the A/D converters, co-adds the A/D output to the previous data and stores the data in RAM, select the next adjacent channel, starts the next A/D conversion, sends a trigger signal to the mirror motor controller if needed, sends a trigger signal to the blackbody motor controller if needed, and sets the transmit flag to "TRUE" if four seconds of integration has been completed. If the transmit flag is "TRUE", the DAS will start transmitting co-added radiometer data, and ancillary data to the CSS. The purpose of placing the acquisition control inside an interrupt is to maintain a constant time interval between the samples. Data transmission can take as long as 300 milliseconds, during this time the acquisition interrupt will pre-empt the transmission and perform its acquisition duties. The execution time of the interrupt is only $300 \mu$ secs. The flowchart and timing diagram for the DAS are shown in Figures 7 and 8 respectively.

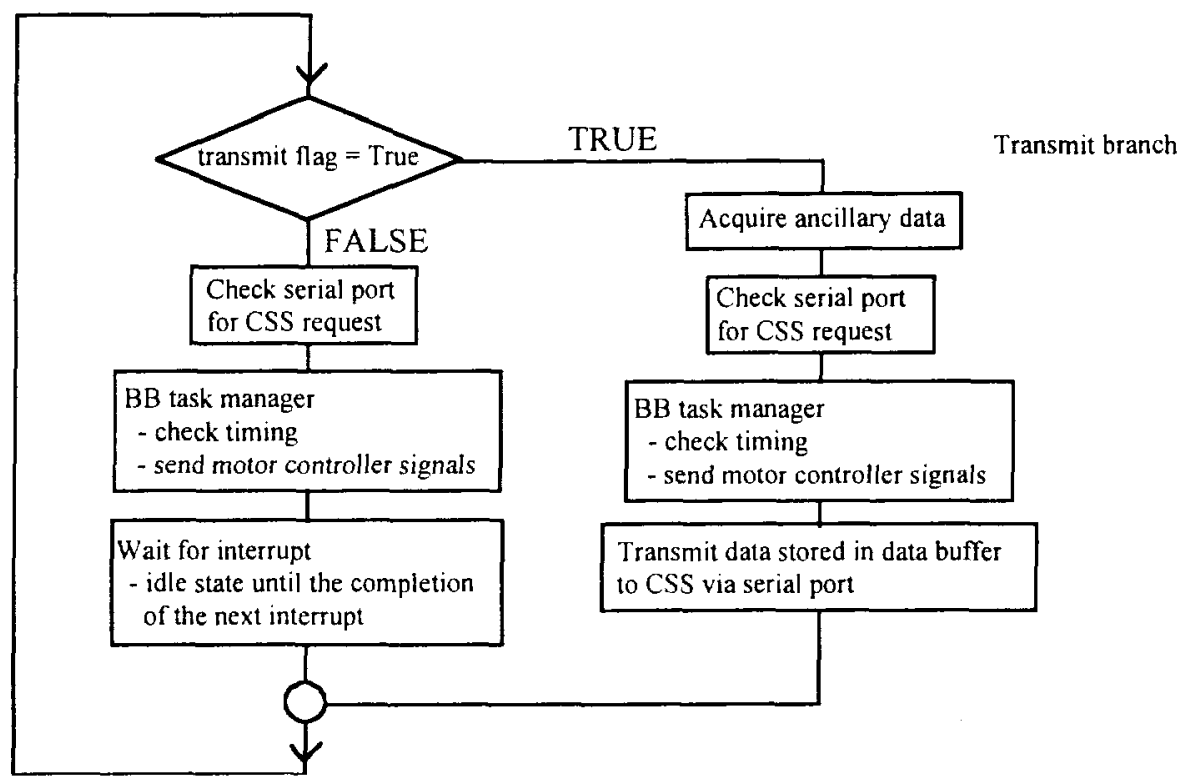

Figure 7. DAS main line flow chart. 


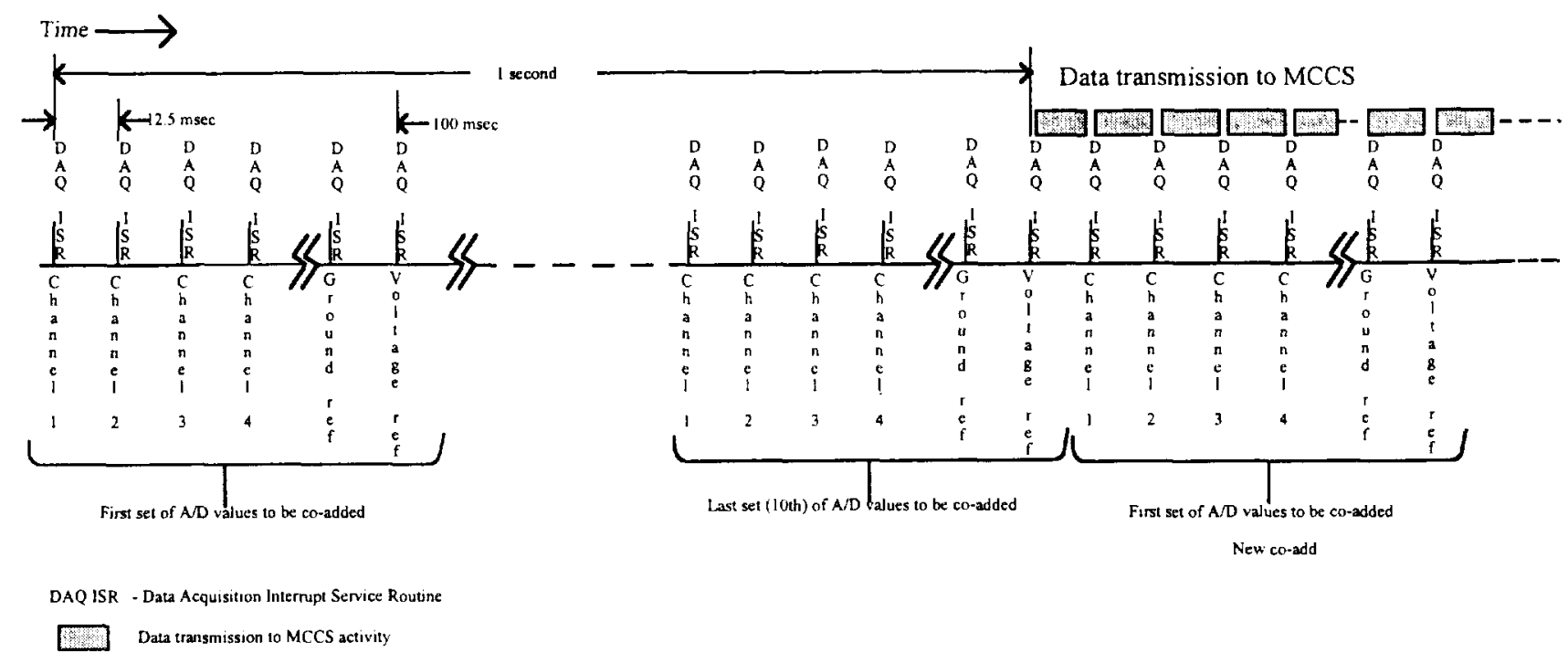

Figure 8. DAS timing diagram.

\section{Control Section Software (CSS)}

The CSS is the component of the WVM that handles commands from the Mission Control Subsystem (MCS) of the aircraft Mission Controls and Communications System (MCCS). The CSS receives commands and data from the MCS through a TCP/IP connection over the local area network of the aircraft. In order to perform the commands, the CSS collects radiometer data and housekeeping data from the DAS through a serial connection.

The two principal commands accepted by the CSS are Calibrate and Operate. The Calibrate command directs the WVM to calibrate its line of sight using aircraft attitude information from the MCS and data from the inclinometer in the WVM. The Operate command tells the WVM to determine the water vapor level above the aircraft in the zenith direction and send that data every 15 seconds to the MCS. For speed and simplicity, the water vapor level is obtained by performing a least-squares fit of the six channels of radiometer data to a pre-computed table of expected radiometer channels values as a function of water vapor level in the atmosphere and the temperature and pressure outside the aircraft.

The CSS software executes on a Themis single board computer running the Solaris operating system, the standard platform chosen for the MCCS. Although Solaris is not a realtime operating system, there was no problem meeting the timing requirements since the data rate from the DAS is not very demanding given the performance of the UltraSPARC processor on the Themis board and the fact that there is no other user process running on the host computer.

There were two main considerations in the design of the CSS: one was the timing requirements and the second was that the WVM is to run autonomously, i.e. without regular human intervention. Since the WVM is designed to run without an operator, there is no need for a user interface, simplifying the CSS software. On the other hand, in addition to the extensive error checking, there is greater emphasis on attempts at error recovery so that the MCS console operator is notified only in case of serious errors where operator action is required. Incidentally, to minimize system administration work, the WVM does not store any water vapor data in the WVMCPU. The tasks of distributing the data to the science instruments and archiving the data are performed by the MCCS.

The CSS software was written in C for speed reasons but uses an object-oriented style for conceptual simplicity. This was possible since there is no graphical user interface and hence no need for a window class library. The use of multiple threads (one for communicating with the DAS, one for communicating with the MCS, and one for computations) greatly simplified the organization of the CSS. 


\section{Instrument Status}

A prototype system consisting of the RHD, six way power splitter, filter network, RF power detector network, data acquisition system for both radiometer and ancillary data, $68 \mathrm{HC} 11$ system under the control of the DAS, and the Themis single board computer system under the control of the CSS, has been constructed and is currently being tested under laboratory conditions. The RHD in this prototype system did not have the mirror assembly or black body units installed so signal chopping was performed manually with external chopper.

The six RF power detector boards were tested for noise, sensitivity, and linearity. The RF signal chain, including the 6way RF power splitter, the six RF amplifiers and filters, and the RF power detector boards, was tested for noise, sensitivity, linearity, and frequency response profile. Finally, an "end-to-end" setup with the RHD connected to the RF signal chain was tested for noise and for response to radiative input chopped manually between ambient $(-300 \mathrm{~K})$ and liquid nitrogen-cooled ( $77 \mathrm{~K}$ ) emitting surfaces.

Typical rms noise levels in the RF chain were in the 1 to $2 \mathrm{mV}$ range. The response of the RF power detector boards for channels one through five were all quite similar, with slopes in the range of about 6.0 to $7.1 \mathrm{mV} / \mathrm{nW}$. With the rest of the RF chain connected (RHD connected to the RF signal chain), the response was similar for channels one through three $(\sim 200 \mathrm{mV} / \mathrm{nW})$, but about a third weaker for channels four and five.

For the end-to-end test, the best noise levels were seen staring at an ambient temperature target, which produced a range of noise values from $0.8 \mathrm{~K}$ to $0.3 \mathrm{~K} \mathrm{rms}$, for channels one and five respectively. These noise levels were obtained for averages of 4-second data subsets, to mimic the planned in-flight data processing flow. For the five working channels, the root-sum-square noise staring at an ambient target was $1.29 \mathrm{~K}$. Signals obtained while alternating between an ambient target and a $77 \mathrm{~K}$ target, as will be done in-flight, had rms noise levels of $0.6 \mathrm{~K}$ to $1.1 \mathrm{~K}$ for channels two to five, and $2.0 \mathrm{~K}$ for channel one. Response to the $220 \mathrm{~K}$ temperature difference between the ambient and cooled targets was in the range of 0.9 to $1.6 \mathrm{mV}$ per $\mathrm{K}$.

The root-sum-square noise of $1.29 \mathrm{~K}$ is significantly higher than the allocated error budget of $0.5 \mathrm{~K}$. However, the prototype system is significantly different from the final flight unit in that no EMI shielding was used in the RF power detector network or in the data acquisition system. The final pieces of the mirror assembly and black body units are in the final phase of fabrication and final assembly of the flight RHD and the IFC electronics is expected by the end of 2002. An RF integrity test will be performed on the final flight unit to check for RF leakage and RF amplifier stability.

\section{ACKNOWLEDGEMENTS}

The authors would like acknowledge the valuable contributions from Regina Flores, Michael Rennick, and Arif Khan for their work in parts procurement, assembly of the WVM system and component characterization. We like to thank Steve Keith and Anna Glukhaya for their early work on the WVM software. We like to thank Jochen Horn for his contribution to the black body unit design and the elliptical mirror design.

\section{REFERENCES}

1. Haas, M. and Pfister, L., PASP, 110, 339, 1998. 